\title{
A cognitive model for routing in agent-based modelling
}

Cite as: AIP Conference Proceedings 2116, 250005 (2019); https://doi.org/10.1063/1.5114245

Published Online: 24 July 2019

Jascha Grübel, Sarah Wise, Tyler Thrash, and Christoph Hölscher

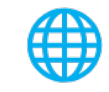

\section{Conference Proceedings}

Get $30 \%$ off all print proceedings!

\section{Enter Promotion Code PDF-30 at checkout}

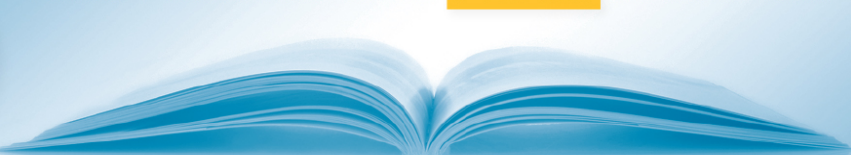




\title{
A Cognitive Model for Routing in Agent-Based Modelling
}

\author{
Jascha Grübel $^{1, a)}$, Sarah Wise ${ }^{2}$, Tyler Thrash ${ }^{1,3,4}$ and Christoph Hölscher ${ }^{1}$ \\ ${ }^{1}$ Chair of Cognitive Science, ETH Zürich, Zürich, Switzerland \\ ${ }^{2}$ Centre for Advanced Spatial Analysis, University College London, London, United Kingdom \\ ${ }^{3}$ Geographic Information Visualization and Analysis, University of Zürich, Zürich, Switzerland \\ ${ }^{4}$ Digital Society Initiative, University of Zürich, Zürich, Switzerland

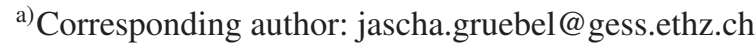

\begin{abstract}
Agent-based modelling (ABM) can be used as a computational tool to model human routing behaviour, and offers particular promise when combined with insights from cognitive science. In this paper, we introduce typical errors into the encoding of the agents mental representation of the environment. This method deviates from the classical computer science paradigm of optimality to capture human behaviour more accurately. By incorporating common distance and direction estimation errors, our model produces routes with fewer computational artefacts such as zigzagging (i.e., turning more often than the typical human) and bottlenecks (i.e., routing through one particular node that maximises efficiency). We demonstrate our results in regular and irregular environments and validate our model using a set of real-world footfall data from Westminster, London.
\end{abstract}

\section{INTRODUCTION}

A route is often considered to be a sequence of intermediate locations between a start location to a goal location [1]. In computer science, routing is formulated as the Shortest-Path problem [2] and classically solved with Dijkstra's Algorithm [3] and successors such as A* [4]. In cognitive science, routing is embedded in wayfinding and involves acquiring, processing, and applying new spatial knowledge [5]. Unlike in computer science, wayfinding is generally not least effort, shortest path or distance minimising [6]. The Shortest-Path Problem on a graph in computer science and the production of a route as application of procedural rules in spatial cognition are indeed homologous. The present approach is to use models from spatial cognition in order to adjust the cost function of the Shortest-Path problem and mimic human behaviour.

According to the spatial cognition literature, human wayfinding involves objectively suboptimal, but subjectively appropriate, decisions. For example, participants consistently underestimate the distance to goal locations [7] and deviate when indicating the direction to goal locations [8]. Such errors are cumulative as more segments along the same route are considered [9]. While human wayfinding is not optimal in the computational sense, it seems that they minimise their effort with respect to a possibly distorted mental representation in order to produce a route [10]. Such a reduction of effort has been described as satisficing [11] or cognitive miserliness [12] and is characterized by matching cognitive effort to the minimal requirements of a task, thus economically balancing cognitive costs with outcome value.

Finding an optimal route between two points A and B is a well-known problem in computer science called the Shortest-Path problem [2] in which the environment is abstracted into nodes (decision points) and edges (distances). Formally, for a graph $G=(V, E)$, a path is defined as a sequence of vertices $P_{v_{1}, v_{n}}=\left(v_{1}, v_{2}, \ldots, v_{n}\right) \in V \times V \times \ldots \times V$ such that there is a connecting edge $e_{i, i+1}=\left(v_{i}, v_{i+1}\right) \in E$ for $1 \leq i \leq n$ between vertices along the sequence. The shortest path $P_{s, t}$ is defined between two vertices $s, t \in V$ such that under an edge weight function $d: E \rightarrow \mathbb{R}$ the sum of weights along the path is minimal, see Eq. 1. For a more detailed discussion, see [13, p. 151].

$$
P_{s, t} \text { s.t. } \underset{e_{i, i+1} \in P_{s, t}}{\arg \min } \sum_{i=1}^{n-1} d\left(e_{i, i+1}\right)
$$

International Conference of Numerical Analysis and Applied Mathematics (ICNAAM 2018)

AIP Conf. Proc. 2116, 250005-1-250005-5; https://doi.org/10.1063/1.5114245

Published by AIP Publishing. 978-0-7354-1854-7/\$30.00 
The main improvements for the classical Shortest-Path solution [3] are grouped together as incremental heuristic search algorithms [2, 14], e.g. A* algorithm [4]. The $\mathrm{A}^{*}$ algorithm adds a heuristic containing "extra knowledge about the problem domain" [15, p. 48] and as such is also a best-first search algorithm. Specifically, a cost function $c_{t}: V \rightarrow \mathbb{R}$ is added to Eq. 1 that approximates the remaining cost to reach the goal vertex $t$. If the heuristic cost function $c$ is consistent or monotone (i.e. it satisfies the inequality $\left.c\left(v_{i}\right) \leq d\left(e_{i, i+1}\right)+c\left(v_{i+1}\right)\right)$ then $\mathrm{A}^{*}$ does not need to revisit nodes to converge. A typical monotone heuristic function is the Euclidean distance.

Although the $\mathrm{A}^{*}$ algorithm does not always produce the best solution, it is the most prevalent in the literature $[16,17]$. Several other solutions to the Shortest-Path problem have been proposed, including variants of $A^{*}[18,19$, 20, 21, 22]), the $\mathrm{D}^{*}$ algorithm family [23, 24], the Bellman-Ford algorithm and derivatives [25, 26, 27, 28], and the Floyd-Warshall algorithm and derivatives [29, 30, 31, 32, 33, 34]. These algorithms are rarely applied to ABM modelling due to their extended complexity.

For the present paper, we apply ABM to test theories of human spatial representation by transforming the graph employed for routing according to common wayfinding errors. We demonstrate that our cognitive routing model (i.e. C*) provides distinct routing behaviour compared to previous Shortest-Path solutions (e.g. A*).

\section{METHODS}

For this paper, we modify the heuristic of the $A^{*}$ Algorithm by replacing the objective criteria (i.e. Euclidean distance) in order to capture cognitive encoding errors while maintaining a minimisation criteria [10]. These errors are based on two key observations. First, the configurational knowledge encodes the relative positions and directions between points to establish a layout [35]. Second, variations in memory are systematic in that experimental observations have shown consistent properties of the error terms. We decompose the error into distance errors and direction errors. Distance errors typically result from perceived distances being shorter than actual distances [36] (see Stevens [37] and Eq. 2 for the formalisation). Empirical estimates of the coefficient are $\beta=0.95 \pm 0.2$ [38, 39]. Direction error has been studied previously (e.g. $[40,41]$ ) but was only recently quantitatively formalised [8] as a circular normal distribution (i.e., [42]). The probability of an agent remembering the correct direction is parametrised as a mean $\mu=0$ and a concentration $\kappa$. When $\kappa=32$ there is a standard error of 30 (cf. [40, 41]). The von Mises distribution is shown in Eq. 3 with $I_{0}$ as the modified Bessel function [43].

These errors are modelled with a two-tiered representation [44] in which reality (facts regarding the environment) is represented separately from the agent's cognition (beliefs regarding the environment). If an agent's beliefs were to match the facts, $C^{*}$ equals $\mathrm{A}^{*}$ in execution. $\mathrm{C}^{*}$ typically differs from $\mathrm{A}^{*}$ as it uses an adapted distance estimation function to represent beliefs. The agent may have incorrect beliefs about the destination (routing towards a different location) or intermediate nodes (routing inefficiently). The influence of these errors decreases as agents approach the destination.

The $\mathrm{C}^{*}$ algorithm is implemented with memory and routing components. The memory component maintains the agents' beliefs regarding known locations at a space cost $O(|V|)$ per agent. For each known location, we shift the coordinates along a polar coordinate system with the agent at its origin. Distance and direction errors are computed based on Eq. 2 and Eq. 3. In Eq. 4, the rotation $\mathbf{R}$ represents a draw from the von Mises distribution. Based on Huttenlocher, Hedges, and Duncan [45], each new draw only contributes at a rate of $\delta=0.2$ to the remembered location, see Eq. 5. The exact values of all parameters were based on the literature but still chosen somewhat arbitrarily as no empirical verification has yet been conducted.

$$
\begin{array}{rcc}
d(x) & = & x^{\beta} \\
p(x \mid \mu, \kappa) & = & \frac{e^{\kappa \cos (x-\mu)}}{2 \pi I_{0}(\kappa)} \\
x^{\prime} & = & \frac{|d(x)|}{|x|} \mathbf{R} \cdot x \\
x_{\text {update }}^{\prime} & = & \delta * x^{\prime}+(1-\delta) x_{\text {old }}^{\prime}
\end{array}
$$

\section{SIMULATION AND RESULTS}

$\mathrm{C}^{*}$ is embedded in the MomenTUM framework for pedestrian ABM [46] and is available at https://github.com/jugdemon/MomenTUM. Agents in MomenTUM have a cognitive model called Spice 


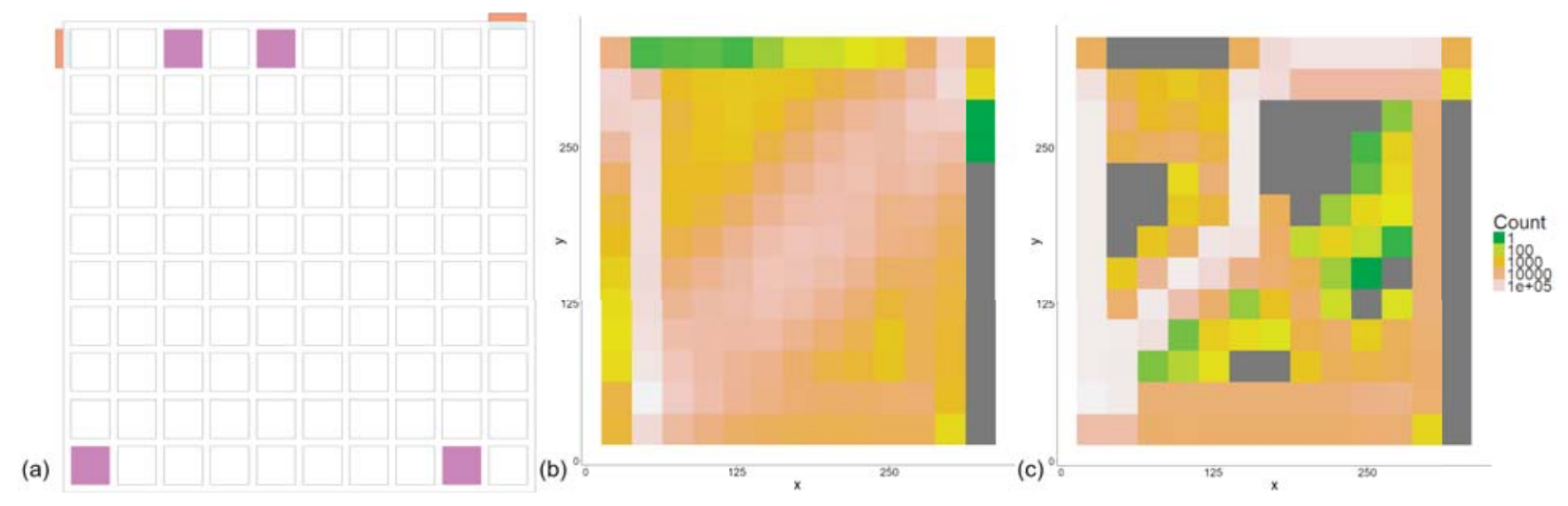

FIGURE 1. Regular Environment - The layout of the environment (a). Heatmaps of the $C^{*}$ agents (b) and $A^{*}$ agents (c).

(i.e. Spatial sequential choice) that explicitly models perception, memory, and interaction with other agents [47]. Our simulations ran for 60 minutes with 1 second time steps and were repeated 1000 times. We compared the routing behaviour between $\mathrm{A}^{*}$ and $\mathrm{C}^{*}$ using regular and irregular layouts, see Figure 1. In addition, we used a layout based on Westminster London to compare the results of $\mathrm{A}^{*}$ and $\mathrm{C}^{*}$ to aggregated footfall data.

The simulations were analysed with respect to 2D-histograms that were generated over each layout and summed over a sample of runs. To evaluate the two-sample comparisons of multivariate data, we used kernel density estimates (KDE) [48]. To test the null hypothesis $H_{0}: f_{1}=f_{2}$, a test statistic is derived from a discrepancy measure [49] comparing the intrasample pairwise differences to the intersample pairwise differences [48]. Rather than relying on overly large samples that increase the sensitivity of statistical tests to noise, Lee et al. [50] suggest to compute the minimum sample size at which properties converge. We determined convergence by varying the number of summed simulations required to robustly identify that two non-overlapping samples with the same routing algorithm belong to the same distribution. We used the minimum sample size 750 to determine that $\mathrm{C}^{*}$ and $\mathrm{A}^{*}$ are not forming the same distribution in all layouts ( $p<=0.046$ ).

The 2D-histogram over the traces of all agents, shown in Figure 1, reveals more diverse route choices with $C^{*}$. Instead of only moving along the shortest path (as with $\mathrm{A}^{*}$ ), the agents more often choose non-optimal routes, but highly unlikely routes are still rarely chosen. In addition, $\mathrm{A}^{*}$ exhibits computational artefacts such as zigzagging (i.e agents turn more than humans) [51, 52]s and bottlenecks (i.e. all agents try to pass through the same minimising point based on diagonal distance instead of a L1 distance (Manhattan Distance) [53]). In general, the $C^{*}$ agents prefer more straight lines. The distortions in their mental representations are large enough to subdue zigzagging and bottlenecks as $\mathrm{C}^{*}$ agents do not account for the minimal improvement provided by the diagonal path. In contrast, $\mathrm{A}^{*}$ agents consider even minimal differences in the configuration space to decide for the optimal route.

We also compared both algorithms to real world data for validation and verification [54]. The data was drawn from the Consumer Data Research Centre's repository of footfall data, collected over a month by SmartStreetSensors. By tracking individual (anonymised) mobile phones, we were able to recreate the flow of individuals between a series of locations. Extracting the entrance and exit locations associated with our samples of individuals, we compared the flow through various intermediate locations and exit locations with that of the real data (results not shown).

\section{DISCUSSION AND FUTURE WORK}

We have shown that $\mathrm{C}^{*}$ agents and $\mathrm{A}^{*}$ agents differ in their behaviour visually as well as statistically. Optimisation artefacts in $\mathrm{A}^{*}$ such as zigzagging and bottlenecks is substantially reduced for $\mathrm{C}^{*}$. This can be ascribed to the difference in memory for the two routing algorithms because $\mathrm{C}^{*}$ is more "fuzzy" and allows agents to "change their mind" in the process of navigating without changing strategy. This contrasts with previous models that only allowed for explicit re-routing based on a general re-evaluation of goals and perception.

$\mathrm{ABM}$ is a useful tool to understand models in cognitive science. Assumptions regarding the mind can be rigorously tested by comparing the behaviour of the model with empirical data [55]. For the present study, we applied theories from cognitive science to propose a more adequate model for simulating human navigation behaviour. The 
work focuses on the process of routing itself and does not include other confounding variables such as learning. We also successfully demonstrated that including an agent's uncertainty in memory into the model produces a more varied result and allows us to more effectively model real behaviour.

This paper suggests many opportunities for further research, both for cognitive science as well as computer science. While the theoretical models used are widely established in cognitive science, implementing them in ABM has exposed several gaps in the literature. ABM has stagnated with a set of classical models that may not hold upon closer inspection, and there is much remaining potential to expand ABM to reflect human behaviour more accurately using input from cognitive science.

\section{ACKNOWLEDGMENTS}

We would like to thank Leonel Aguilar and Dirk Helbing for discussing the mathematical formulation of our model. We would also like to thank Samuel Fux for support with the Euler supercomputer. The validation data for this research have been provided by the Consumer Data Research Centre, an ESRC Data Investment, under project ID CDRC 138, ES/L011840/1; ES/L011891/1.

\section{REFERENCES}

[1] S. Werner, B. Krieg-Brückner, and T. Herrmann, in Spatial cognition II (Springer, 2000), pp. $295-316$.

[2] N. Deo and C.-Y. Pang, Networks 14, 275-323 (1984).

[3] E. W. Dijkstra, Numerische mathematik 1, 269-271 (1959).

[4] P. E. Hart, N. J. Nilsson, and B. Raphael, IEEE transactions on Systems Science and Cybernetics 4, 100-107 (1968).

[5] R. G. Golledge, Wayfinding behavior: Cognitive mapping and other spatial processes 5-45 (1999).

[6] T. Gärling, E. Lindberg, M. Carreiras, and B. Anders, Journal of Environmental Psychology 6, 1-18 (1986).

[7] J. M. Loomis and J. M. Knapp, Virtual and adaptive environments 11, 21-46 (2003).

[8] T. Thrash, H. Zhao, A. Duran, L. Frese, and V. R. Schinazi, Evaluation of a computational framework for cognitive maps. 2017.

[9] T. Hayashi, H. Fujii, and T. Inui, "Modeling the cognitive map formation process based on psychological experiments using a computer graphics system," in Systems, Man and Cybernetics, 1990. Conference Proceedings., IEEE International Conference on (IEEE, 1990), pp. 826-828.

[10] R. G. Golledge, Geoforum 23, 199-214 (1992).

[11] H. A. Simon, Psychological review 63, p. 129 (1956).

[12] N. Barr, G. Pennycook, J. A. Stolz, and J. A. Fugelsang, Computers in Human Behavior 48, 473-480 (2015).

[13] B. Korte, J. Vygen, B. Korte, and J. Vygen, Combinatorial optimization, 4th ed., Vol. 2 (Springer, 2012 ).

[14] S. Koenig, M. Likhachev, Y. Liu, and D. Furcy, AI Magazine 25, p. 99 (2004).

[15] J. Pearl, (1984).

[16] A. T. Crooks and A. J. Heppenstall, in Agent-based models of geographical systems (Springer, 2012), pp. $85-105$.

[17] A. Johansson and T. Kretz, in Agent-based models of geographical systems (Springer, 2012), pp. $451-462$.

[18] R. E. Korf, Artificial intelligence 27, 97-109 (1985).

[19] S. J. Russell, "Efficient Memory-Bounded Search Methods.” in ECAI, Vol. 92 (1992), pp. 1-5.

[20] R. E. Korf, Artificial Intelligence 62, 41-78 (1993).

[21] S. Koenig, M. Likhachev, and D. Furcy, Artificial Intelligence 155, 93-146 (2004).

[22] Y. Björnsson, M. Enzenberger, R. C. Holte, and J. Schaeffer, CIG 5, 125-132 (2005).

[23] A. Stentz and Others, "The focussed D* algorithm for real-time replanning," in IJCAI, Vol. 95 (1995), pp. 1652-1659.

[24] S. Koenig and M. Likhachev, IEEE Transactions on Robotics 21, 354-363 (2005).

[25] A. Shimbel, "Structure in communication nets," in Proceedings of the symposium on information networks, Vol. 4 (1954).

[26] L. R. Ford Jr, "Network flow theory," Tech. Rep. (Rand Corporation, Santa Monica, CA, USA, 1956).

[27] R. Bellman, Quarterly of applied mathematics 16, 87-90 (1958).

[28] E. F. Moore, "The shortest path through a maze," in Proc. Int. Symp. Switching Theory, 1959 (1959), pp. 285-292. 
[29] S. C. Kleene, "Representation of events in nerve nets and finite automata," Tech. Rep. (Rand Corporation, Santa Monica, CA, USA, 1951).

[30] B. Roy, Comptes Rendus Hebdomadaires Des Seances De L Academie Des Sciences 249, 216-218 (1959).

[31] R. W. Floyd, Communications of the ACM 5, p. 345 (1962).

[32] P. Z. Ingerman, Comm. ACM 5, p. 556 (1962).

[33] S. Warshall, Journal of the ACM (JACM) 9, 11-12 (1962).

[34] G. Ramalingam and T. Reps, Journal of Algorithms 21, 267-305 (1996).

[35] R. Briggs, "Cognitive distance in urban space." Ph.D. thesis, The Ohio State University 1972.

[36] A. S. Gilinsky, Psychological review 58, p. 460 (1951).

[37] S. S. Stevens, Psychological review 64, p. 153 (1957).

[38] J. C. Baird and W. R. Biersdorf, Attention, Perception, \& Psychophysics 2, 161-166 (1967).

[39] J. A. Da Silva, The American Journal of Psychology 119-144 (1985).

[40] P. Worchel, Psychological monographs: general and applied 65, p. i (1951).

[41] R. L. Klatzky, J. M. Loomis, R. G. Golledge, J. G. Cicinelli, S. Doherty, and J. W. Pellegrino, Journal of motor behavior 22, 19-43 (1990).

[42] K. V. Mardia and P. E. Jupp, Directional statistics, Vol. 494 (John Wiley \& Sons, 2009).

[43] M. Abramowitz, I. A. Stegun, and Others, Handbook of mathematical functions with formulas, graphs, and mathematical tables, Vol. 9 (Dover, New York, 1972).

[44] H. Hochmair and A. U. Frank, Spatial Cognition and Computation 2, 283-313 (2000).

[45] J. Huttenlocher, L. V. Hedges, and S. Duncan, Psychological review 98, p. 352 (1991).

[46] P. M. Kielar, D. H. Biedermann, and A. Borrmann, TUM-I1643 (2016).

[47] P. M. Kielar and A. Borrmann, "Spice: A Cognitive Agent Architecture for Computational Crowd Simulations in Complex Environments," (2017).

[48] T. Duong, B. Goud, and K. Schauer, Proceedings of the National Academy of Sciences 109, 8382-8387 (2012).

[49] N. H. Anderson, P. Hall, and D. M. Titterington, Journal of Multivariate Analysis 50, 41-54 (1994).

[50] J.-S. Lee, T. Filatova, A. Ligmann-Zielinska, B. Hassani-Mahmooei, F. Stonedahl, I. Lorscheid, A. Voinov, J. G. Polhill, Z. Sun, and D. C. Parker, Journal of Artificial Societies and Social Simulation 18, p. 4 (2015).

[51] H. J. Wiesmann and H. R. Zeller, Journal of Applied Physics 60, 1770-1773 (1986).

[52] M. Kanehara, S. Kagami, J. J. Kuffner, S. Thompson, and H. Mizoguhi, "Path shortening and smoothing of grid-based path planning with consideration of obstacles," in Systems, Man and Cybernetics, 2007. ISIC. IEEE International Conference on (IEEE, 2007), pp. 991-996.

[53] C. C. Aggarwal, A. Hinneburg, and D. A. Keim, "On the surprising behavior of distance metrics in high dimensional spaces," in ICDT, Vol. 1 (Springer, 2001), pp. 420-434.

[54] A. Crooks, C. Castle, and M. Batty, Computers, Environment and Urban Systems 32, 417-430 (2008).

[55] J. P. Davis, K. M. Eisenhardt, and C. B. Bingham, Academy of Management Review 32, 480-499 (2007). 